Meta

Journal des traducteurs

Translators' Journal

\title{
Les principes terminologiques revisités à l'aune des entreprises
}

\section{Sandrine Peraldi}

Volume 60, numéro 2, août 2015

$60^{\mathrm{e}}$ anniversaire. Les horizons de la traduction : retour vers le futur $60^{\text {th }}$ Anniversary. Translation's Horizons: Back to the Future

60mo aniversario. Los horizontes de la traducción: regreso al futuro

URI : https://id.erudit.org/iderudit/1032912ar

DOI : https://doi.org/10.7202/1032912ar

Aller au sommaire du numéro

Éditeur(s)

Les Presses de l’Université de Montréal

ISSN

0026-0452 (imprimé)

1492-1421 (numérique)

Découvrir la revue

Citer ce document

Peraldi, S. (2015). Les principes terminologiques revisités à l'aune des entreprises. Meta, 60(2), 361-361. https://doi.org/10.7202/1032912ar d'utilisation que vous pouvez consulter en ligne. 


\title{
Les principes terminologiques revisités à l'aune des entreprises
}

\author{
Sandrine Peraldi \\ ISIT, Paris, France \\ s.peraldi@isit-paris.fr
}

\begin{abstract}
Puisque «l'invitation est au voyage», cette communication propose de s'intéresser à la manière dont les principes terminologiques, et plus particulièrement, les préceptes définitoires et les relations sémantiques sont aujourd'hui entièrement revisités afin de répondre aux besoins de structuration conceptuelle et d'organisation visuelle de l'information des grandes entreprises. Nous nous appuierons notamment sur une analyse discursive et sémantique menée pour le compte d'une entreprise de conseil en innovation et en ingénierie avancée, dans le domaine de la catégorisation des risques projet.

Cette entreprise, qui accompagne de nombreuses sociétés dans leur processus de développement de nouveaux produits et services, a pour rôle de dimensionner et de piloter les projets qui lui sont confiés, en évaluant leur niveau de criticité. Pour ce faire, elle s'appuie sur un ensemble d'outils et d'indicateurs, parmi lesquels une liste de facteurs de risques plus ou moins hiérarchisée. Cette catégorisation a été établie selon les connaissances a priori et a posteriori et la conceptualisation d'un seul expert, correspondant de fait à une approche ontoterminologique (Roche 2008). Les difficultés d'appréhension et d'application de cette liste de facteurs ont conduit l'entreprise commanditaire à remettre en question cette approche purement conceptuelle en cherchant à la confronter à une approche textuelle, via l'exploitation semi-automatique d'un corpus de textes, afin de dégager les concepts-clés liés aux facteurs de risque et de structurer ces derniers en familles cohérentes et adaptables aux besoins d'un projet.

Nous proposons donc d'exposer notre méthodologie d'analyse du corpus (Bowker et Pearson 2002; L'Homme 2004) et de décomposition des relations sémantiques (Aussenac et Condamines 2007; Barrière 2002). Nous montrerons dans quelle mesure le recours à des théories de l'organisation visuelle de l'information (Matrice de Bertin, Gestalt Theory), d'une part, et aux principes de constitution de la définition canonique (Pavel et Nolet 2001 ; Vézina, Darras et al. 2009), d'autre part, a permis d'accompagner les différentes étapes de recatégorisation et de description conceptuelles des facteurs de risques, dans un effort constant de réconciliation entre approche conceptuelle et textuelle.
\end{abstract}

Titulaire d'un DESS Industrie de la langue et traduction spécialisée et d'un doctorat en linguistique théorique, descriptive et automatique (Université Paris-Diderot), Sandrine Peraldi est Directrice de la recherche à l'ISIT. En charge de la stratégie recherche de l'école ainsi que de la coordination scientifique des projets européens, elle enseigne la terminologie et la linguistique de corpus et dirige l'ensemble des projets de recherche appliquée et des mémoires des étudiants du Master CIT. Auteur d'une trentaine de publications, codirectrice de publication du Bulletin du CRATIL, elle s'intéresse à l'analyse des discours spécialisés et au fonctionnement des termes en contexte par le biais d'approches outillées sur corpus. La traduction automatique et la traduction assistée par ordinateur constituent également un terrain d'investigation privilégié. 